\title{
UNA PROPUESTA DE ANÁLISIS TEXTUAL A PARTIR DE LOS POSTULADOS DEL ANÁLISIS CRÍTICO DEL DISCURSO
}

\author{
Jorge Murillo Medrano \\ Adrián Vergara Heidke
}

\begin{abstract}
RESUMEN
En este trabajo se presenta el modelo de análisis textual provisto por el análisis crítico del discurso (ACD). Se discuten sus principios teóricos y metodológicos para luego aplicarlos a un texto sobre el tema de la transparencia. Esto con miras a despertar el interés de investigadores, en el área de las ciencias sociales, por este modelo de análisis textual.
\end{abstract}

ABSTRACT

This work presents the Model of Textual Analysis provided by the Critical Analysis of the Speech. Its theoretical and methodological principles are described so that they can be thereafter applied to a text dealing with the theme of transparence. The aim is to attract the social science investigators' interest toward this Model of Textual Analysis.

\section{Postulados del análisis crítico del discurso}

A modo de presentación inicial, podemos decir que el análisis crítico del discurso (ACD) corresponde a un particular enfoque del análisis del discurso, el cual se centra en problemas sociales, ocupándose tanto de elementos lingüísticos como de otras dimensiones semióticas.

Su base teórica se origina en pensadores y movimientos sociales y políticos del siglo XX como: el italiano Antonio Gramsci, los filósofos de la Escuela de Frankfurt (Adorno, Habermas, Horkheimer, entre otros), los franceses Louis Althusser, Michel Foucault y Pierre Bourdieu, y el ruso Bajtín'1.

La Escuela de Filosofía de Frankfurt aportó para el desarrollo del ACD, principalmente, el término "crítico" y una concepción particular del papel del lenguaje. Como señala Wodak (2003), crítico (o crítica) debe "entenderse como el resultado de tomar cierta distancia respecto de los datos, enmarcar éstos en lo social, adoptar explícitamente una postura política y centrarse en la autocrítica" (29). Mientras que el lenguaje se considera como un instrumento de dominación, el cual legitima las relaciones de poder por cuanto es ideológico ${ }^{2}$; para ser más precisos, el lenguaje se relaciona con el poder de varias maneras: expresa el poder, moldea el poder y desafía al poder. Esta relación es dialéctica, por cuanto el discurso (lenguaje) 
constituye al poder contribuyendo a sustentar y reproducir el statu quo social, y es constituido por el poder, ya que determina su actualización.

Además de por los distintos enfoques dentro del análisis del discurso, el ACD ha sido influenciado por la "sociolingüística" — al considerar la relación entre el lenguaje y lo social—y, fundamentalmente, por la "lingüística sistémica funcional" (cuyo mayor exponente es M.A.K. Halliday), la cual "[entiende y analiza] al lenguaje como algo configurado [...] por las funciones sociales a las que ha terminado sirviendo" (Fairclough 2003: 186).

\section{Características del ACD}

Hoy en día existen varias líneas del ACD, como la escuela francesa, la lingüística crítica, la semiótica social, el cambio sociocultural y cambio en el discurso, los estudios sociocognitivos, el enfoque histórico del discurso, el análisis de la lectura, la Escuela de Duisburg y el análisis mediato del discurso, por nombrar algunos ${ }^{3}$. A pesar de la gran variedad de posiciones frente al ACD, se puede hablar de ciertas características ${ }^{4}$ generales y comunes de este enfoque lingüístico:

- Se ocupa de problemas sociales, centrándose no sólo en elementos lingüísticos, sino también en otras dimensiones semióticas.

Considera el discurso como una forma de práctica social.

Es interdisciplinario en la teoría, la metodología y el trabajo, por cuanto incorpora teorías y métodos de otras disciplinas (como la filosofía, sociología y psicología) que sean útiles para la investigación.

No existe una teoría ni una metodología capaz de explicar por sí sola el objeto de estudio, por lo cual el ACD recurre a aquellas que serán de utilidad, dentro de todas las posibilidades existentes, siempre en relación con el problema concreto que se vaya a analizar. Por esto se dice que el ACD es multiteórico y multimetódico.

- $\quad$ El ACD toma una postura política explícita con la finalidad de dejar claros (no ocultando) sus elementos y su ideología.

- $\quad$ Es crítico con los problemas sociales y autocrítico con su propio posicionamiento.

- $\quad$ Se posiciona a favor de los grupos dominados.

- $\quad$ El ACD se centra en las relaciones de poder, dominación y desigualdad, en pos de descubrir, revelar y divulgar lo implícito en los discursos, las ideologías que reproducen o resisten al poder.

- $\quad$ Todos los niveles y dimensiones del discurso pueden ser considerados en el ACD.

- $\quad$ El contexto histórico corresponde a un elemento relevante en las investigaciones. 
- $\quad$ Otro aspecto a considerar es la intertextualidad e interdiscursividad de los discursos abordados.

- $\quad$ El ACD tiene un objetivo práctico, ya que busca que los resultados obtenidos puedan ser aplicados para cambiar los problemas investigados. Además, intenta que los estudios no se queden en oficinas y bibliotecas de especialistas, sino darlos a conocer a la población en general, por lo cual deben ser accesibles para la mayoría tanto en lenguaje como en divulgación.

A partir de estas características, podemos decir que el ACD corresponde a un enfoque interdisciplinario dentro del Análisis del Discurso, el cual se ocupa de problemas sociales -como las relaciones de poder, dominación y desigualdad—, con el fin de descubrir, revelar y divulgar las ideologías que reproducen o resisten al poder, por medio de una multiteoría y multimetodología, tomando una postura política explícita y una perspectiva tanto crítica como autocrítica.

\section{Ideología, poder, dominación y discurso}

Dentro de la base teórica del ACD, hay ciertos conceptos recurrentes como ideología, poder, dominación y sus relaciones con el discurso, los cuales desarrollaremos a continuación.

\subsection{Ideología}

El término ideología se entiende, frecuentemente, como un sistema de ideas, representaciones, actitudes, el cual determina a un individuo o a un grupo de personas. Esta concepción posiciona a la ideología en el ámbito de lo "ideal", del "mundo de las ideas", sin establecer un nexo con lo material (realidad física). Ante esta situación, Althusser (1970) propone una nueva definición, relacionando la ideología con las prácticas sociales y con la constitución del sujeto. La ideología, según este autor, "tiene una existencia material" (1970: 57), por cuanto aquélla es en los actos, existe en ellos. Si una persona realiza un acto, lo hace de una determinada manera de acuerdo con su ideología; además, ese acto se inserta en una práctica social, la cual está regulada por rituales definidos por la ideología. Es decir, cada acto es ideológico, debido a que no puede, de ninguna forma, realizarse aisladamente de la ideología propia del sujeto. Por lo anterior, Althusser concede ese carácter "material" a la ideología, dejando de lado la concepción "ideal". Cabe señalar que para este autor existe una gradación de lo "material”; así, la ideología es material, pero de un distinto nivel que lo es una mesa o una silla.

Otro aspecto corresponde a la relación ideología-sujeto. En este caso, para que haya ideología necesariamente tiene que existir un sujeto concreto, porque aquélla es propia de los individuos, entendidos como seres reales que realizan actividades materiales. En otras palabras, sin sujeto no hay ideología. Sin embargo, esta relación no se queda ahí solamente, ya que la ideología constituye al sujeto, por cuanto no es posible la existencia de un sujeto sin una ideología. En resumen: 
-No hay práctica [social] sino por y bajo una ideología.

-No hay ideología sino por el sujeto y para los sujetos (Althusser 1970: 63).

De esta manera, podemos decir que la ideología corresponde a un conjunto de ideas, representaciones, actitudes, el cual constituye a los individuos y es constituido por ellos, además de determinar y existir en las prácticas sociales de éstos, teniendo siempre una existencia material.

\subsection{Poder, dominación y hegemonía}

El concepto de poder que desarrollaremos, sigue los planteamientos de Michel Foucault, el cual señala: "El término "poder" [...] incluye un gran número de mecanismos individuales, definibles y definidos que parecen capaces de inducir conductas o discursos" (citado en Jäger 2003: 100). Es decir, el poder corresponde al conjunto de mecanismos, algunos ya definidos y otros capaces de serlo, los cuales poseen la potencialidad de lograr que se realicen determinadas acciones (conductas y discursos); constituye una estrategia, "plan de prácticas más o menos preciso e [...] intencional [...] que se adopta con el fin de alcanzar un determinado objetivo social, político, psicológico o lingüístico" (Wodak 2003: 115). Esta estrategia no se posee, no es algo inherente a un individuo o grupo de personas, sino que se ejerce, invadiendo a los que no lo tienen, lo cual conlleva cierta movilidad, en cuanto no es propiedad o elemento constitutivo de un sector y puede, en un momento dado, ser ejercido por un grupo diferente. Esto último determina su carácter tenso, ya que los distintos sectores sociales intentan poder ejercer el poder (valga la redundancia). Para Foucault (1995: 33-34), las relaciones de poder (luchas de poder) se dan en lo profundo de la sociedad, no simplemente en las relaciones Estado-ciudadanos ni en las clases.

El concepto de poder se encuentra íntimamente relacionado al de ideología, ya que, por un lado, este último es constitutivo del sujeto (y constituido por el sujeto) y existe en las prácticas sociales, y, por otro lado, el poder corresponde al ejercicio de una estrategia o plan de prácticas; es decir, responde y manifiesta una ideología en esos actos (insertos en las prácticas). El poder es ideológico, y, al llevarlo a cabo, busca establecer su ideología sobre otras.

Aquí, aparece el término dominación, el cual se refiere a que un grupo determinado de personas ejerce su poder, es decir, establece su ideología mediante coerción sobre otro sector social para alcanzar un fin ${ }^{5}$ determinado, provocando que este sector actúe conforme a la ideología dominante. Hay muchos mecanismos de dominación, unos explícitos, por la fuerza, y otros implícitos en las prácticas sociales cotidianas, los cuales se encuentran ocultos o se presentan como verdades indudables; entre ellos está el discurso.

Cuando la dominación ha llegado a niveles en los cuales los distintos grupos no perciben la base ideológica, la hacen parte de su sentido común o la ubican más allá incluso, entonces hablamos de hegemonía. Antonio Gramsci (1967) señala que la hegemonía "encierra y presupone unidad intelectual y ética [además de económica y política] conforme a una concepción de lo real que ha superado al sentido común" (74). Es decir, el grupo dominante ha ejercido de tal manera su poder que los otros sectores han asimilado como suya la ideología de los otros y actúan inconscientemente de acuerdo con ella. La hegemonía generalmente se establece por medio de los mecanismos implícitos de dominación, ya que difícilmente se lograría mediante la fuerza. 


\subsection{Discurso}

El discurso lo entendemos como "una práctica social, [...] una forma de acción entre las personas que se articula a partir del uso lingüístico contextualizado, ya sea oral o escrito" (Casimiglia 1999: 15). A partir de esta definición, se observa que el discurso corresponde a una práctica social. Recordemos que nuestra concepción de ideología (siguiendo a Althusser) señalaba que la ideología existía en las prácticas sociales, que éstas se dan por y bajo una ideología, a partir de lo cual podemos enunciar: hay discursos por y bajo una ideología y en ellos existe una ideología; por lo tanto, son ideológicos. Ahora bien, el lenguaje constituye uno de los elementos de mayor relevancia en las sociedades: por medio de él nos comunicamos, transmitimos las tradiciones y los saberes, entre otras cosas; además, participa de otras prácticas no propiamente discursivas. Jürgen Habermas (1982: 179) al respecto señala que "la gramática del lenguaje ordinario no establece solamente relaciones inmanentes al lenguaje, sino que regula el entramado comunicativo de enunciados, acciones y vivencias en su totalidad", y agrega, "fija esquemas de concepción del mundo y de interacción" (1982: 195). Esta capacidad del lenguaje (discurso) de establecer una concepción del mundo, entendida como ideología, y de relacionarse (o participar) de casi todas las demás prácticas, es lo que provoca que el discurso corresponda a uno de los principales mecanismos para ejercer el poder, ya que mediante él se puede dominar e, incluso, lograr una hegemonía.

Los distintos sectores intentan, a través del discurso, ejercer su poder y establecerse como grupo dominante; por lo tanto, en el discurso se puede observar la lucha de poderes entre ellos. Sin embargo, por el carácter social de esta particular práctica, es decir, que todos los sectores poseen un dominio (manejo) del lenguaje, el mecanismo no es explícito, sino implícito, lo cual dificulta su percepción y facilita la posibilidad de que el grupo dominante vaya manipulando a los dominados, de manera que su ideología vaya asimilándose, estableciéndose como natural, para, así, lograr la hegemonía. Ante esto es que el ACD se centra en esas luchas y relaciones de poder y dominación, con el fin de descubrir, revelar y divulgar lo implícito en los discursos por medio del análisis de los distintos elementos lingüísticos presentes en los objetos de estudios.

\section{Metodología de análisis}

El ACD, como señalamos anteriormente, no posee una metodología de análisis preestablecida, sino que selecciona la teoría y los métodos según el objeto de estudio en concreto. Aunque pudiera parecer que existe una variedad muy heterogénea de formas de analizar los textos, pues ello depende de la naturaleza de los mismos discursos que se analizan, los principios generales que siguen los diferentes lingüistas que han trabajado en esta línea son bastante similares. Meyer (2003) se aboca a la tarea de describir las diferentes posiciones metodológicas que se han empleado en la tradición del análisis crítico del discurso y plantea características generales que tipifican los diferentes acercamientos.

En primer lugar, aclara que se asume que todos los discursos son históricos, es decir, que solo pueden ser entendidos y analizados por referencia a su contexto. Este contexto incluiría aspectos sociopsicológicos, políticos e ideológicos (lo cual le imprime una característica interdisciplinaria al análisis), además de que toma en cuenta los conceptos de intertextualidad 
e interdiscursividad; es decir, analiza cómo se inscriben otros textos y discursos en el texto que se analiza. Las relaciones lengua y sociedad no se asumen como directas, causales o efectistas sino que se plantea la existencia de mecanismos de mediación: Fairclough (1995), por ejemplo, echa mano de los conceptos de la gramática sistémica de Halliday y del orden del discurso de Foucault para explicar estas mediaciones, en tanto que van Dijk (2001), defiende la existencia de procesos sociocognitivos como mediadores de esta relación.

Otra característica importante de la metodología del ACD, según Meyer, es la incorporación de categorías lingüísticas específicas al análisis: estas pueden ser desde categorías discursivas como el tema y el rema, el montaje de los textos; pragmáticas, como el estudio de la deixis o de las presuposiciones e implicaturas; hasta más formales, como el estudio del léxico, de las estructuras morfosintácticas o bien, en discursos orales, de aspectos fonéticos.

En tercer lugar, apunta Meyer (2003) que “...el ACD considera, por regla general, que su procedimiento es un proceso hermenéutico, aunque esta característica no sea completamente evidente en la posición que adoptan algunos autores" (p.38), en tanto que el análisis que hace el ACD de los textos trata de encontrar y aprehender las relaciones significativas partiendo de las partes para comprender el texto como un conjunto.

En cuanto a la recolección de los datos, es importante destacar el hecho de que el ACD no ha definido, a la fecha, una manera exclusiva para llevar a cabo tal labor. Si se leen algunos ejemplos de análisis llevados a cabo por algunos autores dentro de la tradición del ACD, en ocasiones ni siquiera hay una referencia específica a cómo se obtuvieron los datos o si éstos, por ejemplo, pertenecen a un corpus más extenso. Meyer señala, a este respecto, que

\footnotetext{
...no se considera que la obtención de los datos sea una fase específica que deba haberse completado antes de comenzar el análisis: tras el primer ejercicio de recogida de datos, lo que procede es efectuar los primeros análisis, hallar indicadores para conceptos concretos, elevar los conceptos a categoría, y, sobre la base de estos resultados, recoger nuevos datos (muestreo teorético) (48).
}

En general, puede afirmarse que los autores que han realizado trabajos de análisis de textos dentro del ACD han tomado los datos de textos televisivos, textos de prensa, actas parlamentarias, conversaciones grabadas y textos en Internet, por ejemplo.

Específicamente en cuanto a los pasos seguidos para el análisis de los textos, hay varias sugerencias, aunque aquí nos limitaremos a presentar la de Van Dijk (2001) no porque sea necesariamente la mejor, sino porque consideramos que se adecua mejor para el modelo de análisis que presentamos. Este autor sugiere que en el análisis deberían tomarse en cuenta aspectos como los siguientes:

El análisis de las macroestructuras semánticas: esto es, de los temas y de las macroproposiciones.

El análisis de los significados locales, lugares en el que las muchas formas de significado tácito o indirecto, como las implicaciones, las presuposiciones, las alusiones, las ambigüedades, las omisiones y las polarizaciones resultan especialmente interesantes. - $\quad$ El análisis de las estructuras formales "sutiles": aquí es donde se analizan la mayoría de los marcadores lingüísticos como el orden y la entonación, el orden de las palabras, el estilo léxico, la coherencia, las iniciativas semánticas locales, la elección del tema, 
los actos de habla, la organización esquemática, las figuras retóricas, las estructuras sintácticas, las estructuras proposicionales, los turnos de palabra, las objeciones y los titubeos.

- $\quad$ El análisis de las formas o los formatos del discurso global y local.

- $\quad$ El análisis de las realizaciones linguiísticas específicas, como las hipérboles, las lítotes, etc.

- $\quad$ El análisis del contexto.

\section{Un ejemplo de análisis textual}

El texto que hemos elegido para presentar el análisis apareció publicado como editorial del periódico La Nación, el 17 de setiembre del 2003. Nos llamó la atención puesto que hemos venido trabajando con un corpus más amplio que está constituido por diferentes textos periodísticos en los que se menciona el concepto de transparencia en noticias o comentarios asociados con el dominio político costarricense. Aunque este concepto aparece en diferentes alusiones de notas periodísticas, en esta ocasión constituyó el tema central del editorial del periódico, lo cual evidencia, a todas luces, un interés particular por destacar esta noción como medular en la escena política. Transcribimos el texto para una mejor comprensión del análisis:

\section{Amagos de transparencia}

\section{- No se combate la corrupción con una ley de prensa obsoleta}

Pese a las exigencias de los electores y la retórica de los políticos, la transparencia en Costa Rica es una asignatura pendiente. La prueba no está solamente en los escándalos constantes, sino en lo poco que se hace para evitarlos. Todo lo publicado sobre el financiamiento ilegal de las campañas electorales no basta para que la Asamblea Legislativa se decida a reformar la ley, llena de portillos por donde ingresan al proceso político torrentes de contribuciones cuestionables.

El plan contra la corrupción enviado al Congreso por la Contraloría General de la República viene dando tumbos desde 1999. La semana pasada, el proyecto quedó condenado al archivo porque los diputados oficialistas rehusaron prolongar su permanencia en la corriente legislativa. La reacción de las fracciones de oposición obligó a los socialcristianos a reconsiderar el punto, y su jefe de bancada ofreció promover una nueva votación. El proyecto castiga el tráfico de influencias, el soborno internacional y el enriquecimiento ilícito, al tiempo que crea nuevos mecanismos de control, como la declaración de bienes de los diputados. La Contraloría envió la propuesta al Congreso porque la ley vigente, como ocurre con el Código Electoral, contenía vicios de inconstitucionalidad que la fueron desmembrando hasta convertirla en un texto inútil, sin capacidad alguna de coerción. Estas reformas, que contribuirían a fortalecer la capacidad del Estado para combatir 
la corrupción, requieren el complemento de una legislación de prensa moderna, que abra el debate público y permita a los ciudadanos ejercer su derecho a fiscalizar el manejo de los asuntos de la colectividad.

Pero en esta materia, la Asamblea Legislativa también está morosa. La Comisión creada para analizar las reformas al régimen jurídico de la información sesiona desde hace tres años y, salvo algunas excepciones, el trabajo realizado demuestra solo desconocimiento del tema, falta de estudio y obstinada resistencia a crear mecanismos de fiscalización social. Para llegar a soluciones idóneas, habría bastado con que la Comisión estudiara la legislación y jurisprudencia de otros países democráticos, pero algunos diputados decidieron dar rienda suelta a su creatividad, como si se tratara de un tema inédito en el mundo. El resultado es un manojo de mociones que anulan los avances pretendidos cuando el Congreso creó la Comisión. En un caso, los diputados establecen el principio del reportaje neutral existente en países como España y Argentina. Ese principio permite a los informadores eximirse de responsabilidad al reproducir manifestaciones de terceros responsables e identificados. La idea es que, cumplidos ciertos requisitos, la responsabilidad sea de quien da las declaraciones y no de quien las transmite. Punto y seguido, los diputados intercalan una frase que anula la anterior, porque dice que la exención de responsabilidad solo opera si las declaraciones consignadas no son un delito contra el honor.

Así las cosas, los congresistas autorizan a los informadores a consignar citas inocuas, que no acarreen responsabilidad para nadie. Es decir, los autorizan a hacer lo que nadie puede prohibirles. No se trata de instar a los diputados para que legislen de manera precipitada. Por el contrario, la experiencia de las leyes contra la corrupción y el Código Electoral demuestran los peligros de aprobar normas mal pensadas. Sin embargo, en todos estos casos han pasado los años sin que el país pueda señalar esfuerzos capaces de demostrar que en materia de transparencia hay algo más que amagos.

Como primer paso del análisis, procedimos a establecer las macroestructuras semánticas del texto. Éstas normalmente corresponden con las ideas básicas que articulan el tejido semántico, es decir, corresponden con la armazón conceptual del todo. En el caso anterior, establecimos las siguientes macroproposiciones:

I. macroproposición: La transparencia es una asignatura pendiente en Costa Rica, a pesar de las exigencias de los electores y de la retórica de los políticos.

$1 \quad$ II. macroproposición: El plan contra la corrupción, enviado por la Contraloría General de la República, ha venido dando tumbos en el Congreso, con lo cual tampoco se ha aprobado una legislación de prensa moderna.

$1 \quad$ III. macroproposición: No ha habido voluntad, en la Asamblea Legislativa, para aprobar una legislación de prensa adecuada. 
IV. macroproposición: Se concluye, entonces, que en materia de transparencia no hay más que amagos.

Tal y como lo plantean Brown y Yule (1983: 87), la linealidad del texto exige leer siempre a partir de lo que viene en primer lugar; es decir, lo primero siempre impone una interpretación a lo que se afirma luego. En este caso, el texto abre con una frase concesiva que incluye a dos actores del espacio político: los electores y los políticos, los cuales vienen asociados, a su vez, con dos sustantivos abstractos: en el primer caso, se relacionan con exigencias; en el segundo, con retórica. En términos de análisis discursivo, diríamos que estos elementos constituyen el tema (o tópico) del primer sintagma y lo segundo (que la transparencia es una asignatura pendiente), el rema. El montaje total del texto se hace, pues, a partir de estos dos elementos y se esperaría que, en un nivel general, en el texto se enfrenten estos dos actores y sus acciones, dado que se establece una antinomia (exigencias $\neq$ retórica) entre los términos con ellos asociados. Sin embargo, tomando en cuenta el resto de las macroproposiciones, nos damos cuenta de que surge un tercer actor en el discurso: la prensa, la cual entra en oposición con el dominio de lo político y, por ende, se establece una afiliación entre prensa y electores. Perfilada esta homologación, en los tres párrafos restantes se contraponen, entonces, las acciones de los políticos y los deseos de una ley de prensa moderna para luchar contra la corrupción.

Tomando como base lo anterior, se comprende mejor el título del editorial y su subtítulo: en realidad, el texto tiene como propósito fundamental abogar por la creación de una nueva ley de prensa (pues la actual es obsoleta) con la cual se aseguraría una transparencia en el actuar de los políticos. Al respecto de los elementos que componen el título (un programador de lectura), hay que señalar que son dos sintagmas en relación de determinación: amagos de transparencia. El primero de ellos se define como señal de algo, o bien como intento fallido. No obstante, debemos considerar que las palabras no solo tienen el significado básico dado por el diccionario, sino que están cargadas de valores semánticos recogidos de las distintas formaciones discursivas por las que han pasado. En este caso particular, el término amago se ha empleado frecuentemente, en nuestro medio, asociado con la esfera del enfrentamiento político y se ha acuñado incluso una frase al respecto: amagos de violencia. Se puede plantear, de esta manera, que existe una referencia exofórica que se integra al título, se deconstruye y se pone a funcionar en un nuevo escenario textual: el de la transparencia. A partir de lo anterior, suponemos que la semiosis del texto se instaura sobre dos ejes: intentos fallidos y transparencia. Analizaremos, a continuación, cómo se articulan, en el tejido discursivo, estas dos esferas semánticas con sus respectivos actores.

Iniciamos con el segundo elemento, la transparencia, el cual se supone constituye el eje del texto. Esperaríamos, según una primera impresión, que se constituya un campo semántico de términos asociados con el dominio de la transparencia, empleando para ese fin sinónimos dominados por el hipónimo, transparencia. El primero de ellos que emplea es asignatura pendiente: con ese recurso metafórico se trae a colación el discurso pedagógico y se instaura así un espacio en el que la transparencia se concibe como parte de unos deberes, los cuales deben ser cumplidos por un grupo que aprende en un salón de clase. El parangón es bastante obvio, pues se metaforiza la Asamblea Legislativa con tal entorno. Los actores, entonces, verían minimizado su papel de legisladores al de alumnos o aprendices y, lo que es peor, estudiantes que no han cumplido con sus deberes. La posición del que escribe, por ende, no será 
entonces el del ente objetivo que denuncia la falta de legislación al respecto, sino el de un ojo avisor que reprende por no haber cumplido con la tarea escolar.

Sin embargo, el mecanismo léxico semántico más utilizado para constituir ese campo semántico es el uso de la antonimia: el dominio de la transparencia se define a partir de sus contrarios: escándalos constantes, financiamiento ilegal de las campañas electorales, torrentes de contribuciones cuestionables, para tomar solo algunos ejemplos del primer párrafo. Es interesante notar cómo las sustituciones léxicas antonímicas van desde lo más general, los escándalos constantes, los cuales podrían ser interpretados por el lector como posibles en distintos contextos, hasta lo más particular y restringido: lo relativo a las campañas electorales, con lo cual se dirige intencionalmente al lector a reinterpretar los escándalos en el ámbito mucho más delimitado de la política electoral.

El segundo párrafo no hace referencia ya a la transparencia sino que inicia con un sustituto léxico bien definido: el plan contra la corrupción. Se sobreentiende, a estas alturas del desarrollo discursivo, que este plan combate la corrupción y, por lo tanto, es un plan en pro de la transparencia. Con él, se eliminarían el tráfico de influencias, el soborno internacional y el enriquecimiento ilícito. Nótese, por otra parte, cómo liga el editorialista los nuevos mecanismos de control que se propiciarían con este nuevo plan al ámbito específico de los diputados, pues arguye que éstos permitirían ejercer control sobre, por ejemplo, la declaración de bienes de los diputados.

Tomando como base los escuetos ejemplos anteriores, puede colegirse que la corrupción es el quid del asunto (y no necesariamente la transparencia, como se anuncia en el título del editorial), y que esta se encuentra íntimamente relacionada con un grupo de actores textuales (y políticos) específicos: los diputados. Esto se ha anunciado desde el primer enunciado de todo el texto: la retórica de los políticos.

Pasando a un segundo ámbito léxico semántico, tendríamos entonces el de la no corrupción, es decir, el de la transparencia como tal. Los mecanismos léxicos utilizados para definir este campo semántico son diversos: el primero de ellos consiste en oponer al grupo de diputados con la exigencia de los electores. Es importante destacar el uso del término exigencia, pues esta idea aparecerá, matizada de diversas formas, a lo largo del texto. La segunda vez que aparecen los electores como actores textuales se recurre a un sustituto léxico: los ciudadanos. Esta vez se presentan como fiscalizadores de la función pública: requieren el complemento de una legislación de prensa moderna, que abra el debate público y permita a los ciudadanos ejercer su derecho a fiscalizar el manejo de los asuntos de la colectividad. En este enunciado, no obstante, se establece además una identificación entre los ciudadanos, como actores, y los periodistas. Por medio de una presuposición se aduce que, si se tiene una legislación de prensa moderna, los ciudadanos podremos fiscalizar el manejo de nuestros asuntos y, por ende, el actuar de los diputados.

Respecto de lo anterior, podemos ir desenmascarando cómo se articulan los actores o sujetos del discurso en el texto anterior: el que escribe, el periodista o el informador, aboga por una ley de prensa contra la corrupción (es decir, a favor de la transparencia) y, en ese sentido, se identifica con el pueblo (los electores, los ciudadanos) para exigirles transparencia a los diputados, a los cuales, a su vez, les son asignadas cadenas de sustituciones léxicas (por metáfora o metonimia) alusivas a actos de corrupción, tráfico de influencias y mal manejo de los asuntos públicos.

Volviendo al primer componente léxico del título, amagos, tenemos que también se asocia el campo semántico por él constituido con el accionar de los congresistas: viene 
dando tumbos, la Asamblea Legislativa también está morosa, el trabajo realizado demuestra solo desconocimiento del tema, falta de estudio y obstinada resistencia, avances pretendidos, legislen de manera precipitada y normas mal pensadas. Todos los sintagmas anteriores apuntan a una consideración negativa del trabajo de los congresistas (para cuya descripción, por cierto, se echa mano de términos del discurso pedagógico, para ser coherente con la asociación hecha en el primer párrafo del texto). Es decir, entonces, que los diputados conformarían el grupo de actores políticos que no les permitirían al pueblo y a la prensa ejercer una función fiscalizadora y aseguradora de la transparencia.

En el cuadro siguiente, se pueden visualizar mejor las relaciones establecidas entre las macroestructuras semánticas del texto, los actores textuales y los atributos a ellos referidos:

\section{Cuadro 1}

Eje de la transparencia

-exigencia de los electores

-asignatura pendiente

-plan contra la corrupción

-legislación de prensa moderna

Actores: los electores (el pueblo) y los informadores
Eje de la no-transparencia (amagos de transparencia)

-retórica de los políticos

-financiamiento ilegal de campañas electorales

-torrentes de contribuciones cuestionables

-Asamblea Legislativa morosa (desconocimiento

del tema, falta de estudio y obstinada resistencia)

Actores: los diputados (tratados como escolares que no han cumplido con sus deberes)

Según el esquema anterior, es clara la relación maniquea que se establece: por un lado, en el bando de los buenos, de los que buscan la transparencia, se encuentran los informadores y el pueblo, identificados por sus intereses comunes de luchar contra los malos, los diputados, grupo al que se asocia con un sinnúmero de características negativas.

El binomio anterior se ve reforzado en el estilo léxico que usa el escritor del texto cuando se refiere a ambos dominios:

$-$

Para los electores y los informadores, como grupo, encontramos lexemas como: exigencias, prensa moderna, debate público, fiscalizar, asuntos de la colectividad, informadores, electores y ciudadanos.

- Para los diputados, tenemos: escándalos, portillos, torrente, dar tumbos, morosidad, dar rienda suelta, manojo de mociones, citas inocuas, normas mal pensadas, entre otros.

Vale la pena rescatar dos aspectos, de entre los varios que se podrían comentar. En primer lugar, la autorreferencia que hace el editorialista a todo el gremio de periodistas como informadores. Bien sabemos que el lexema periodista está cargado de semas asociados con la política, también; pero en este caso, se prefiere el término informadores, más neutral a ese respecto y que, en principio, describiría un trabajo más impersonal y objetivo. En segundo lugar, el pueblo aparece tipificado por dos referencias concretas a sus obligaciones políticas: electores y ciudadanos. No se caracterizan como personas del común, sino que se echa mano a esos dos lexemas que los representan dentro de la escena política. 
Por otra parte, los lexemas referidos al ámbito de los diputados se seleccionan de un lenguaje más coloquial, mucho más descriptivo y cargado de semas negativos: torrentes, portillos, dar rienda suelta, por ejemplo.

Según el somero análisis anterior, se evidencian los intereses de un grupo en relación con los de otro: en este caso particular, los periodistas (ahora informadores), representados por el editorialista de este diario (por cierto, uno de los de mayor circulación en el país), se nos presentan como un grupo profundamente interesado en la lucha contra la corrupción, es decir, un gremio que aboga por la transparencia en el ejercicio político. Para ello, lían sus intereses con los del pueblo y se identifican plenamente con los electores. No es gratuita la topicalización que se hace en el nivel macrotextual: lo primero que aparece son las exigencias de los electores, aunque luego solo se mencione a los ciudadanos una sola vez en el texto. Es evidente que los electores (ciudadanos) solo se utilizan como pretextos para atacar al grupo de diputados, a los cuales se les representa casi como "satanizados" por el conjunto de acciones malvadas que llevan a cabo. La transparencia sería, así, una característica típica de los informadores, que solo buscarían una mejor fiscalización en aras del bien de la colectividad.

En el texto, se representa una lucha de dos grupos de poder: el de los periodistas y el de los diputados, con la desventaja para los segundos de que ellos no tienen acceso a los medios de comunicación en la forma en que lo tienen los primeros. El pueblo, el tercer grupo actor que aparece en el texto, no tiene nada que ver: solo se usa como artificio para la argumentación discursiva del editorialista, como una asociación necesaria para hacerse ver como si pertenecieran al grupo de los desposeídos (de poder) cuando, en realidad, es todo lo contrario.

Por último, debe mencionarse el hecho de que no es gratuito que se asocie a los informadores con la lucha por la transparencia. Este concepto, de buen arraigo ya en nuestras sociedades capitalistas, surge asociado con el de globalización. La noción de transparencia permea ahora nuestra sociedad en casi todos los niveles, desde la pequeña empresa hasta la institución estatal. Es un requisito sine qua non para aquellas sociedades que quieran entrar en el proceso de modernización y en el mundo globalizado y, precisamente, serían los periodistas los que enarbolen la bandera de la lucha contra la corrupción en aras de la transparencia nacional.

\section{A modo de conclusión}

Hemos pretendido, en este trabajo, mostrar cuáles son los principios fundamentales del análisis crítico del discurso, como un modo de análisis textual nacido en el seno de la intersección entre varias disciplinas: la lingüística, la sociología, la psicología, la antropología y la filosofía, entre otras. Aunque en principio ha sido practicado por lingüistas, esta manera de analizar (e interpretar textos) se presta para que cualquier investigador de las ciencias sociales pueda también utilizar las herramientas en su trabajo de análisis textual. Se discutieron, además, los principios metodológicos y se presentó un ejemplo, aunque escueto, de aplicación práctica del ACD, con el propósito de que despierte el interés de otros investigadores en el campo. 


\section{Notas}

1. Para una descripción más detallada, ver Fairclough y Wodak 2000, "Análisis crítico del discurso".

2. Los conceptos "ideología”, "poder”, “dominación”, "hegemonía” y "discurso” se desarrollarán más adelante.

3. Ver Wodak y Meyer 2003. Métodos de análisis crítico del discurso y Fairclough y Wodak 2000. “Análisis crítico del discurso".

4. Ver Wodak 2003."El enfoque histórico del discurso” y Van Dijk 1997. Racismo y análisis crítico de los medios.

5. No nos referimos a un único fin determinado, sino a un conjunto de fines en distintas áreas; por supuesto que un sector social puede desear ejercer su poder sobre todas las áreas habidas; en tal caso sí se podría hablar de un único fin global.

\section{Bibliografía}

Althusser, Louis. 1970. Ideología y aparatos ideológicos de estado (notas para una investigación). México: Quinto Sol.

Brown, Gillian y George Yule. 1993. Análisis del discurso. Madrid: Visor.

Casamiglia, Helena y Amparo Tusón. 1999. Las cosas del decir. Barcelona: Ariel.

Fairclough, Norman. 1995. Critical discourse analysis: the critical study of language. London: Longman.

2001. Language and Power. London: Longman.

2003. "El análisis crítico del discurso como método para la investigación en ciencias sociales". En Wodak, Ruth y Michael Meyer (Comps.), 179-203

Fairclough, Norman y Ruth Wodak. 2000 “Análisis crítico del discurso". En Van Dijk, Teun, 367-404.

1995. Vigilar y castigar: nacimiento de la prisión. Traductor Aurelio Garzón del Camino. México: Siglo Veintiuno.

Foucault, Michel. 2002. El orden del discurso. Traductor Alberto González Troyano. Barcelona: Tusquets.

Gramsci, Antonio. 1967. La formación de los intelectuales. Traductor Ángel González Vega. México: Grijalbo. 
Habermas, Jürgen. 1966. Teoría y praxis. Ensayos de filosofía social. Traductor D.J. Vogelmann. Buenos Aires: Sur.

1982. Conocimiento e interés. Traductor Manuel Jiménez, José F. Ivars y Luis Martín Santos. Madrid: Taurus.

1989. Ciencia y técnica como «ideología». Madrid: Tecnos.

Jäger, Siegfried. 2003. "Discurso y conocimiento: aspectos teóricos y metodológicos de la crítica del discurso y del análisis de dispositivos". En Wodak, Ruth y Michael Meyer (Comps.), 61-100

Meyer, Michael. 2003. "Entre la teoría, el método y la política: la ubicación de los enfoques relacionados con el ACD”. En Wodak, Ruth y Michael Meyer (Comps.), 35-59.

Van Dijk, Teun. 2001. "Principles of Critical Discourse Analysis." En Wetherell, M. et al. (Comps.), 300-317.

2000. El discurso como interacción social. Barcelona: Gedisa.

Wetherell, Margaret; Stephanie Taylor y Simeon Yates (Comps.). 2001. Discourse Theory and Practice. London: Sage Publications.

Wodak, Ruth y Michael Meyer (Comps). 2003. Métodos de análisis crítico del discurso. Traductores Tomás Fernández y Beatriz Eguibar. Barcelona: Gedisa.

Wodak, Ruth. 2003. "De qué trata el análisis crítico del discurso (ACD). Resumen de su historia, sus conceptos fundamentales y su desarrollo". En Wodak, Ruth y Michael Meyer (Comps.), 17-34.

2003. "El enfoque histórico del discurso". En Wodak, Ruth y Michael Meyer (Comps.), 101-203. 